\title{
Competencia vectorial de las especies de Lutzomyia del grupo verrucarum (Diptera, Psychodidae) en un foco endémico de Leishmania braziliensis en Reventones, Cundinamarca
}

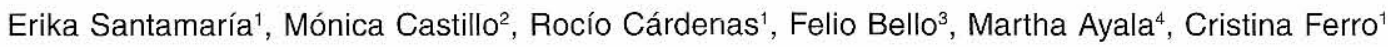

\begin{abstract}
Resumen
Cinco grupos de hembras silvestres de Lutzomyia del grupo verrucarum (Lutzomyia torvida, Lutzomyia ovallesi y Lutzomyia nuñeztovari) presentes en la zona cafetera de Reventones, Cundinamarca, donde Leishmania braziliensis es endémica, se infectaron experimentalmente con el fin de determinar la susceptibilidad a infectarse y la capacidad para transmitir el parásito por picadura a un hospedero. Las hembras de las tres especies, reunidas en la misma jaula, se alimentaron a través de membrana de piel de pollo con una mezcla de promastigotes de cultivo de L. braziliensis y eritrocitos humanos lavados. El porcentaje de infección se determinó por observación directa al microscopio de formas flageladas en los intestinos de las hembras disecadas, entre 4 y 7 días después de la comida infectiva. En los dos primeros grupos, se registró únicamente la susceptibilidad de estas especies a infectarse con L. braziliensis. En los tres grupos restantes, además de observar la susceptibilidad, las hembras alimentadas que sobrevivieron a la oviposición se alimentaron por segunda vez en hámster sano con el fin de intentar la transmisión del parásito por picadura. Las tasas de infección generales obtenidas en los cinco grupos estuvieron entre 23,9 y $70,2 \%$. Las tres especies se infectaron experimentalmente con $L$. braziliensis, siendo $L u$. torvida la más abundante en los ensayos, seguida de Lu. ovallesi. Lu. nuñeztovari se encontró presente en la mayoría de los grupos en una densidad muy baja. No se detectó la presencia de la forma amastigota en ninguno de los hámsteres analizados. Estos resultados indican que las tres especies son susceptibles a infectarse con L. braziliensis, que pueden estar involucradas en el ciclo de transmisión del parásito en el foco de Reventones, Cundinamarca, y por ahora se consideran como vectores potenciales.
\end{abstract}

Palabras clave: infección experimental, transmisión experimental, grupo verrucarum, Leishmania braziliensis

Vectorial competence of Lutzomyia species from the verrucarum (Diptera, Psychodidae) group found in a Leishmania braziliensis endemic focus in Reventones, Cundinamarca

\section{Abstract}

Five groups of wild Lutzomyia females from the verrucarum group, Lutzomyia torvida, Lutzomyia ovallesi and Lutzomyia nuñeztovari, captured in a mountainous coffee-grow-

\footnotetext{
1 Laboratorio de Entomología, Instituto Nacional de Salud, Santafé de Bogotá, D.C., Colombia.

2 Departamento de Química y Biología, Universidad de la Salle, Santafé de Bogotá, D.C., Colombia.

3 Laboratorio de Entomología, Biología Celular y Genética, Universidad de la Salle, Santafé de Bogotá, D.C., Colombia.

4 Laboratorio de Parasitología, Instituto Nacional de Salud, Santafé de Bogotá, D.C., Colombia.

Recibido marzo 19 de 1999, aceptado junio 18 de 1999
} 
ing site in Reventones (Cundinamarca) where Leishmania braziliensis is endemic, were experimentally infected in order to determine their susceptibility to infection with this parasite and their ability to transmit the parasite to a hamster by biting. All three sand fly species (placed together in the same cage) were fed using chicken membrane with a mixture of cultured promastigotes and human erythrocytes. In the first two groups, only the sandfly's susceptibility to infection by the parasite was registered. In the other three groups (besides susceptibility to infection) the females which survived to oviposition were allowed to feed on a hamster in order to transmit the parasite by bite. Infection percentage was calculated from direct observation of the parasite's flagellate form in the gut of dissected females 4 to 7 days after ingesting the infected meal. General infection rates for the five groups ranged from $23.9 \%$ to $70.2 \%$. The three species were susceptible to L.braziliensis infection. In each group Lu.torvida was the most abundant species, followed by Lu.ovallesi. Lu.nuñeztovari was present in low density in all groups. The parasite's amastigote form was not observed in any of the hamsters. These results demonstrate that the three species are susceptible to developing and harbouring L.braziliensis in their gut and that all of them could be involved in this parasite's transmission in the endemic disease focus in Reventones (Cundinamarca) and that they can at least be considered as being potential vectors.

Key words: experimental infection, experimental transmission, verrucarum group, Leishmania braziliensis

\section{Introducción}

La importancia médica de los flebótomos, dípteros hematófagos de la familia Psychodidae, radica principalmente en el papel vectorial que estos pequeños insectos desempeñan en la transmisión de protozoarios del género Leishmania, agentes etiológicos de diferentes formas clínicas de leishmaniasis (1). Se acepta generalmente con raras excepciones, que Leishmania braziliensis Vianna 1911 es el parásito responsable del desarrollo de la leishmaniasis mucocutánea (2), el cual se caracteriza por presentar metástasis de la infección, particularmente en la región de las fosas nasales, la mucosa oral y la faríngea donde produce lesiones de carácter destructivo (3).

Esto constituye un serio problema de salud pública en Latinoamérica (4). En Colombia y Venezuela, esta enfermedad se ha encontrado asociada principalmente con áreas montañosas donde se cultiva el café (5-9). En estos países, generalmente la fauna de flebótomos en las plantaciones cafeteras, incluye como mínimo una de las especies del grupo verrucarum (10), considerado de especial importancia en salud pública ya que la mayoría de sus especies son altamente antropofílicas y varias de ellas son vectores probables o confirmados de Leishmania (11). Dicho grupo está conformado a su vez por las series: townsendi, verrucarum y serrana (12). De la serie townsendi, dos miembros han sido reportados naturalmente infectados con parásitos de L. braziliensis: Lutzomyia spinicrassa Morales et al. 1969 en Arboledas, Norte de Santander (5) y Lutzomyia youngi Feliciangeli \& Murillo en Trujillo, Venezuela (8). Lutzomyia longiflocosa Osorno-Mesa et.al. 1970, también de la serie townsendi, es considerada el más probable vector de $L$. braziliensis en el foco endémico de Tello-Baraya, Huila (13). De la serie verrucarum, Lutzomyia evansi (Nuñez-Tovar 1924) fue encontrada naturalmente infectada con Leishmania chagasi Cunha y Chagas 1937 en un foco de leishmaniasis visceral en Colombia (14); Lutzomyia ovallesi (Ortiz 1952) ha sido incriminada como vector de $L$. braziliensis en Guatemala (15) y Venezuela (16), en Bélice, Panamá y Colombia ha sido hallada con flagelados no identificados $(5,17)$ y en Panamá es considerada como vector sospechoso de Leishmania panamensis Lainson \& Shaw 1972 (18). Lutzomyia nuñeztovari anglesi Le Pont Desjeux 1984, también de la serie verrucarum, es el probable vector de leishmaniasis cutánea en el foco de Yungas, Bolivia, donde es el único 
flebótomo antropofílico capturado en el intradomicilio; además, durante 1982 y 1983 , varios especímenes de $L u$. $n$. anglesi del mismo foco, fueron encontrados con promastigotes en posición peripilórica lo cual sugiere que el parásito podría ser del complejo Leishmania Vianna (V) (19). Recientemente, Torrez et al. confirmaron la infección natural de esta especie de Lutzomyia con un parásito perteneciente al complejo Leishmania Vianna mediante técnicas de biología molecular (20).

Entre 1986 y 1993, se aisló y tipificó el parásito L. braziliensis de seis pacientes procedentes de la zona rural de la Inspección de Policía de Reventones, municipio de Anolaima, Cundinamarca, región considerada como foco endémico de leishmaniasis cutánea y mucocutánea (datos no publicados, Laboratorio de Parasitología, INS). Estudios entomológicos puntuales realizados en la misma época, determinaron la presencia en el foco de tres especies de Lutzomyia del grupo verrucarum: $L u$. torvida Young, Morales \& Ferro 1994, Lu. ovallesi y Lu. nuñeztovari (Ortiz 1954). Lu. torvida, perteneciente a la serie townsendi, representa la especie antropofílica más abundante en esta zona (21); su descripción original se realizó precisamente con material recolectado en estos estudios e inicialmente, mientras se comprobó que representaba una nueva especie fue llamada Lutzomyia sp. de Reventones (22). Hasta la fecha sólo se ha registrado en esta Inspección y, como antecedente vectorial, está el hallazgo en 1987 de un ejemplar naturalmente infectado con promastigotes que no se lograron aislar ni tipificar (23). Las otras dos especies, pertenecientes a la serie verrucarum como se mencionó, son reconocidas por su importancia médica y en Colombia presentan una amplia distribución, $L u$. ovallesi en los departamentos de Antioquia, Boyacá, Caldas, Cundinamarca, Magdalena, Norte de Santander y Santander (24) y Lu. nuñeztovari a lo largo de la Cordillera Oriental compartiendo su hábitat con otras especies de la serie townsendi en Cundinamarca, Huila (25), Norte de Santander (26) y Tolima (27).

Las tres especies en el foco de Reventones, se han registrado en el extra, peri e intradomicilio; en este último, Lu. torvida presenta la mayor densidad mientras que $L u$. ovallesi y Lu.nuñeztovari están presentes en una proporción más baja. Esto indica que las tres especies están en contacto con la población humana, por lo cual podrían estar involucradas en la transmisión de $L$. braziliensis (datos no publicados, Laboratorio Entomología, INS). Es importante llevar a cabo estudios que ayuden a establecer el papel como vector de estas especies en dicho foco y, de esta forma, mejorar el conocimiento de la epidemiología de la enfermedad y contribuir en el diseño de métodos para un futuro control del vector.

Según Killick-Kendrick (28), dos de los criterios importantes en la incriminación de una especie de flebótomo como vector comprobado de Leishmania son: el insecto debe permitir la multiplicación del parásito en su intestino y, además, debe ser capaz de transmitirlo a un hospedero reservorio por picadura. Los estudios de infección y transmisión experimental son un acercamiento para mejorar el entendimiento sobre los ciclos de transmisión de Leishmania en la naturaleza (29). En el laboratorio, se pueden efectuar ensayos que permiten medir la susceptibilidad de una especie de flebótomo a infectarse con determinada especie de Leishmania, por medio de infecciones experimentales que facilitan la observación del desarrollo y multiplicación del parásito en su posible vector; a su vez, se pueden obtener hembras potencialmente infectadas que se alimenten por segunda vez sobre hámster sano y así observar la habilidad de estas especies para transmitir el parásito a un hospedero reservorio por picadura (30).

En este trabajo se evaluó la susceptibilidad a la infección con L. braziliensis de tres especies de flebótomos del grupo verrucarum ( $L u$. torvida, $L u$. ovallesi y $L u$. nuñeztovari) presentes en el foco endémico de esta especie de Leishmania en Reventones, Cundinamarca, y se realizaron ensayos de transmisión del parásito a hámsteres sanos por picadura de hembras potencialmente infectadas de las tres especies.

\section{Materiales y métodos}

Flebótomos. Las recolecciones de los flebótomos se llevaron a cabo entre septiembre de 1997 y 
septiembre de 1998, en la vereda El Platanal, perteneciente a la Inspección de Policía de Reventones, municipio de Anolaima, Cundinamarca, localizada a los $04^{\circ} 47^{\prime} 35^{\prime \prime}$ de latitud norte y $74^{\circ} 29^{\prime} 35^{\prime \prime}$ de longitud occidental, sobre el flanco occidental de la Cordillera Oriental a una altitud de $1.500 \mathrm{msnm}$ con una temperatura promedio de $24{ }^{\circ} \mathrm{C}$ y correspondiente a una zona cafetera. Estas capturas se realizaron en cafetales cercanos a las viviendas, de las $18: 30$ a las $22: 30$ horas, utilizando trampas Shannon y cebo humano protegido (figura 1a). A medida que los insectos se iban capturando, se colocaron en una jaula de muselina de $20 \times 20 \mathrm{~cm}$ para ser posteriormente alimentados. En dicha jaula se reunieron todos los flebótomos capturados por noche.
Parásitos. Los promastigotes de $L$. braziliensis (MHOM/CO/86/CL-250) (MHOM/BR/75/CL-813) se mantuvieron en medio de Schneider en una concentración de $1-2 \times 10^{6}$ promastigotes por mililitro. En el momento de la infección, este medio se mezcló con eritrocitos humanos lavados, en una proporción 1:1.

Procedimiento de infección. Terminada la captura, se procedió a alimentar las hembras a través de membrana de piel de pollo de 1-3 días de edad, siguiendo el método descrito por Tesh y Modi, 1984 (31); para esto, esa misma noche, se colocó en la parte superior de la jaula, un recipiente cubierto con la delgada membrana la cual sostenía la solución de parásitos; dicho recipiente era rodeado con los dedos protegidos de uno de los integrantes del equipo de trabajo con el fin

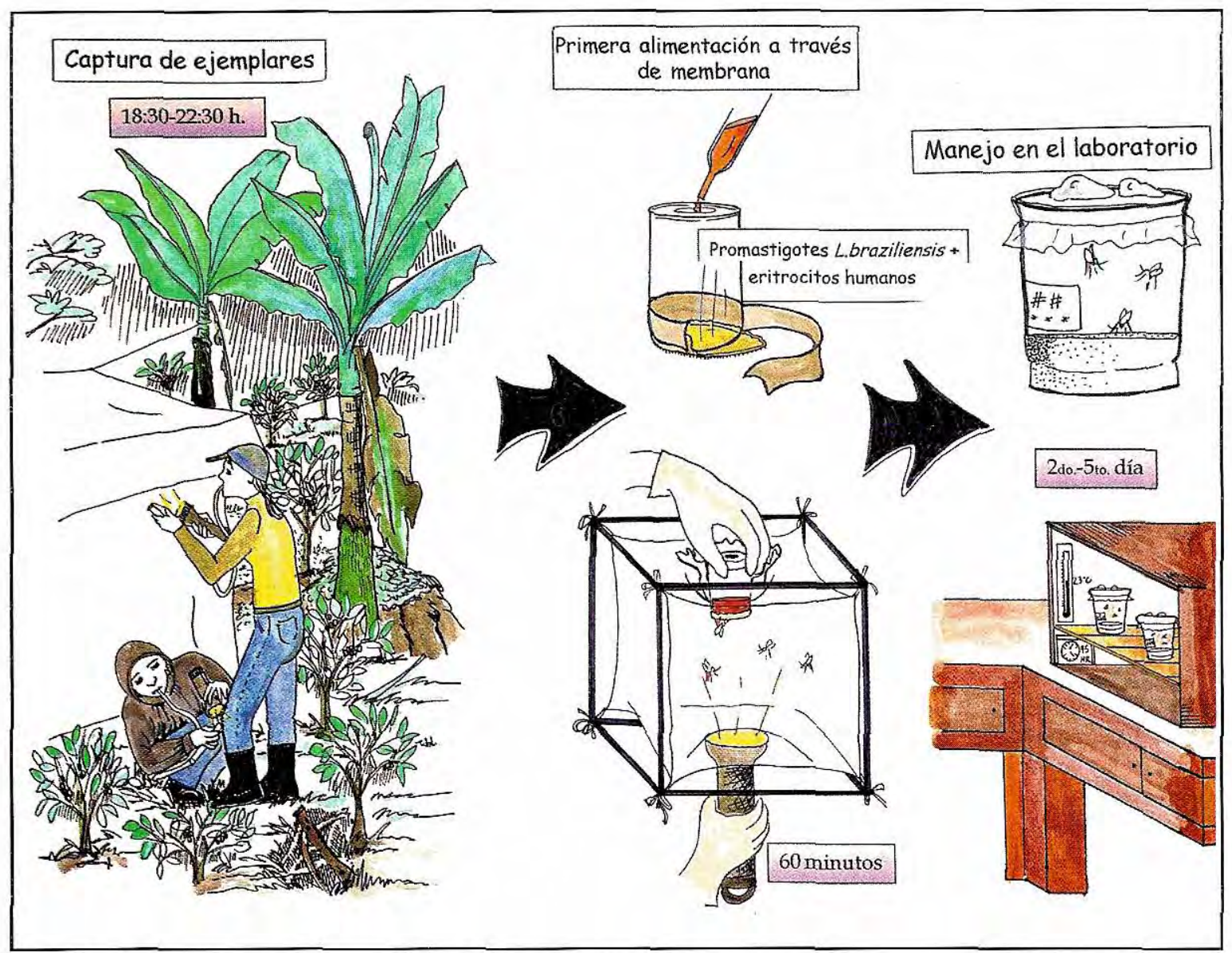

Figura 1a. Captura, infección y manejo de los flebótomos en el laboratorio. 
de estimular los insectos a alimentarse del vial. También se ubicó una linterna en la parte inferior de la jaula de tal forma que se iluminara el recipiente con la comida infectiva y las hembras se dirigieran allí, ya que se ha observado en los estudios de campo realizados en este foco, que las especies antropofílicas son atraidas por la luz (datos no publicados, Laboratorio de

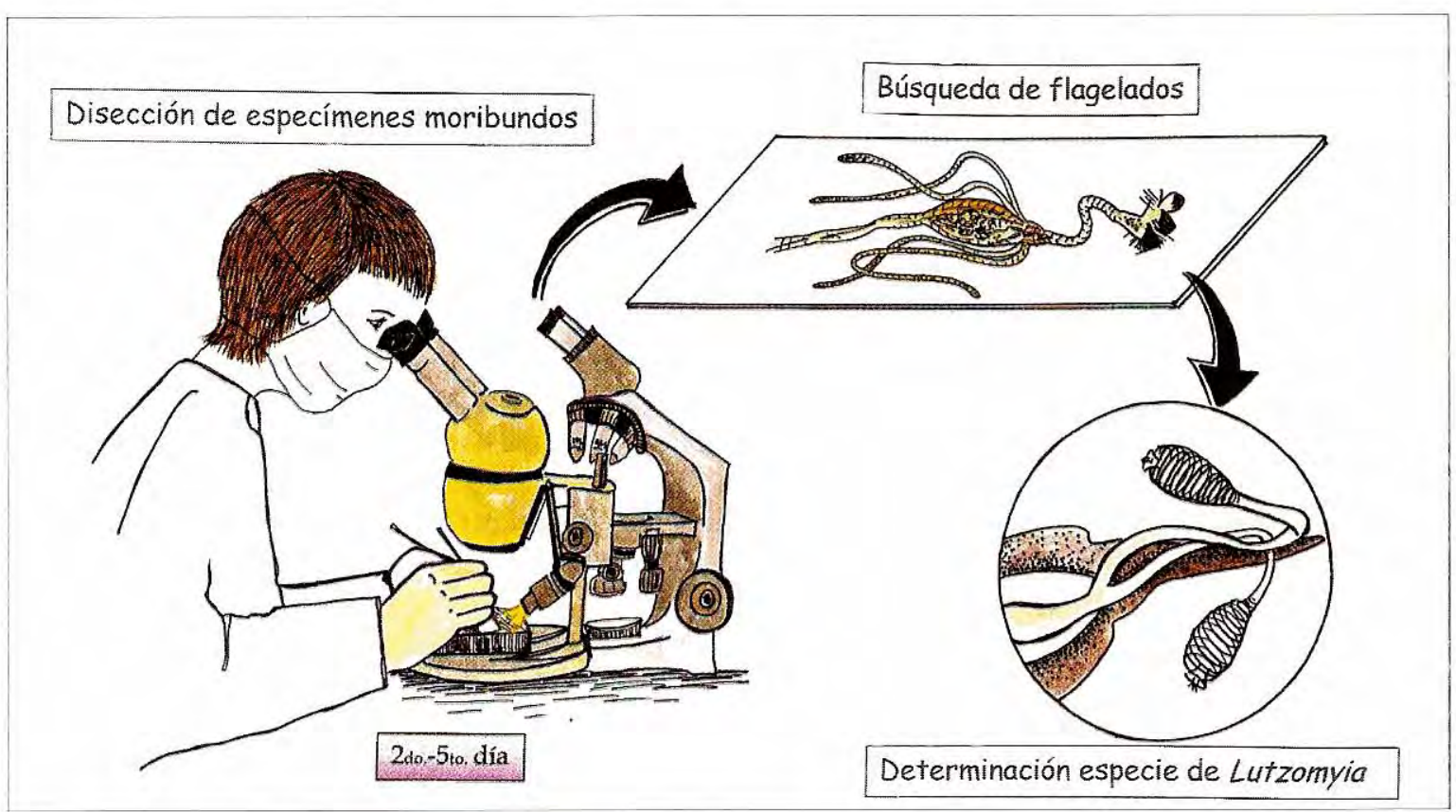

Fibura 1b. Disección y búsqueda de promastigotes

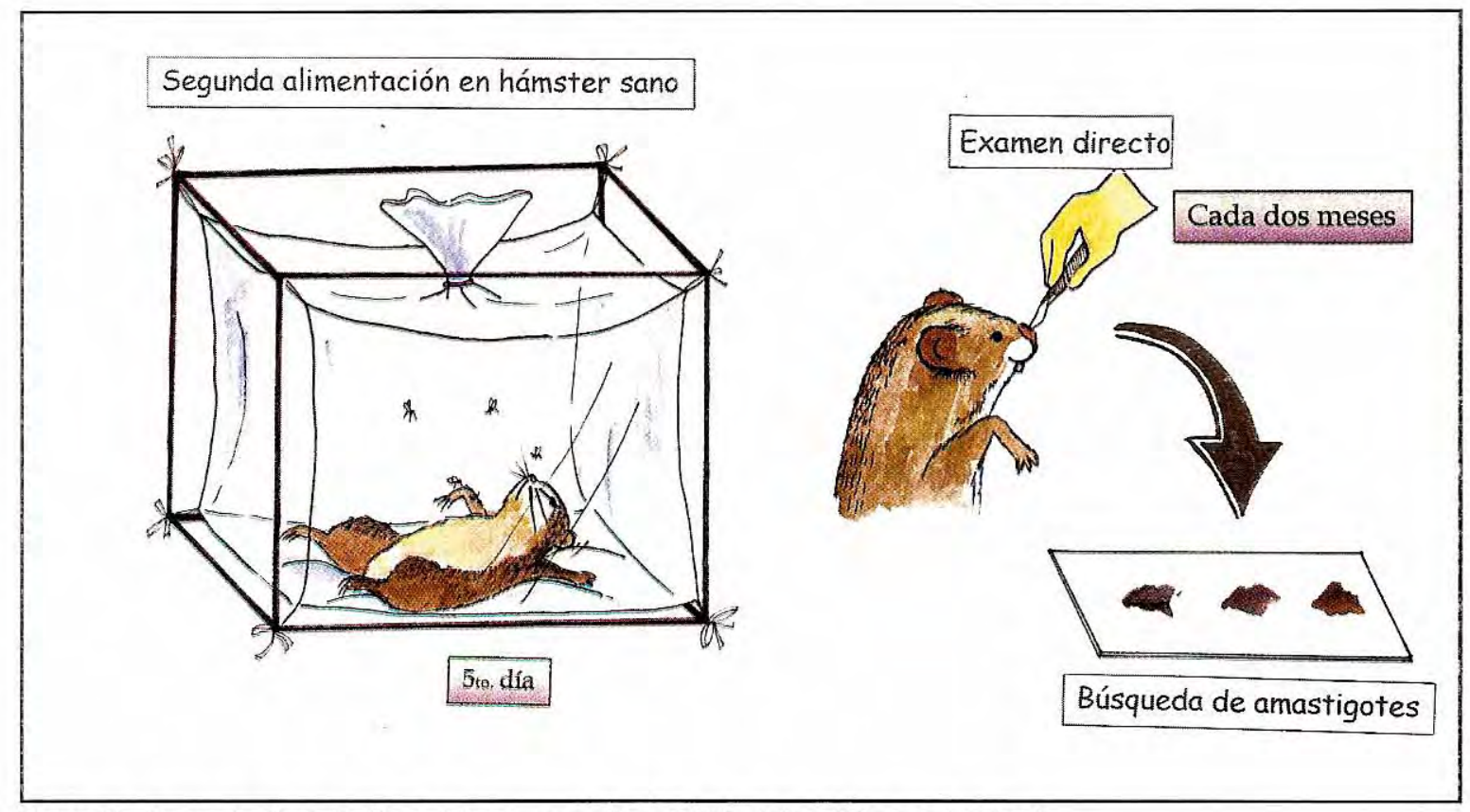

Figura 1c. Segunda alimentación en hámster sano (transmisión del parásito por picadura). 
Entomología, INS). Posteriormente, las hembras que se alimentaron fueron separadas en vasos plásticos y transportadas al insectario del Instituto Nacional de Salud en Santa Fe de Bogotá, donde se mantuvieron a una temperatura promedio de $23{ }^{\circ} \mathrm{C}$ y humedad relativa de $95 \%$, con una dieta de agua y solución azucarada (13) (figura 1a).

Infección y transmisión. Se conformaron 5 grupos; cada uno de ellos correspondió a una captura diferente, en distintos meses del año. En los dos primeros grupos únicamente se registró la susceptibilidad del insecto a la infección; para esto, las hembras se disecaron entre 4 y 7 días después de la comida infectiva, en solución salina con ayuda de un estereoscopio, extrayendo el tracto digestivo completo para búsqueda de formas flageladas (promastigotes) bajo el microscopio de luz (figura $1 \mathrm{~b}$ ).

Se determinó el porcentaje de hembras infectadas y se registró la distribución y densidad de los promastigotes. En los tres grupos restantes, las hembras que sobrevivieron al sexto día después de la comida infectiva, se expusieron a una segunda alimentación sobre hámster sano con el fin de observar si esta especie era capaz de transmitir el parásito a un hospedero por picadura (figura 1c). En los dos días siguientes, se disecaron las hembras realimentadas para observar si estaban o no infectadas y, en caso positivo, registrar las características de la infección. En cada una de las hembras disecadas, se examinaron las espermatecas para confirmar la especie de Lutzomyia (13) (figura 2).

Hámsteres picados por hembras de Lutzomyia potencialmente infectadas. Estos hámsteres fueron revisados semanalmente y pasados dos y cuatro meses de haber sido picados, se les realizaron exámenes directos tomando improntas en láminas de muestras de linfa de nariz, patas traseras y delanteras y coloreando con Giemsa para la búsqueda de amastigotes bajo el microscopio (figura 1c).

\section{Resultados}

Los porcentajes totales de infección de las hembras alimentadas con solución de parásitos de $L$. braziliensis a través de membrana en cada uno de los cinco grupos fueron de 57,1, 23,9,
$70,2,45,2$ y $31,6 \%$, respectivamente (cuadro 1 ). En las disecciones se encontró un alto número de promastigotes en el intestino posterior, especialmente en la región del píloro, observándose menos parásitos en el intestino medio y anterior. En algunas hembras de $L u$. torvida infectadas, se observaron formas de cuerpo corto, flagelo largo y alta movilidad, consideradas como formas infectivas, las cuales se encontraban ubicadas en el intestino anterior. No se detectó actividad de los promastigotes en la faringe, cibarium o proboscis de los insectos.

En el cuadro 1 se aprecia que las especies de Lutzomyia capturadas y alimentadas con parásitos a través de membrana, corresponden a tres especies del grupo verrucarum: $L u$. torvida, Lu. ovallesi y Lu. nuñeztovari. Lu. torvida, con excepción del grupo 3, predominó en cuanto a abundancia y número de hembras que presentaron infección. En el tercer grupo, $L u$. ovallesi fue la especie más abundante y la que presentó mayor porcentaje de infección y, en general, fue la segunda especie más frecuente en las capturas. Lu. nuñeztovari estuvo presente e infectada en la mayoría de los grupos aunque en una proporción mucho más baja.

Los promastigotes en los intestinos de las hembras infectadas, inicialmente se localizaron en el píloro, con una migración leve hacia el intestino medio y anterior; esto coincide con la clasificación que hace Lainson (32) de las especies de Leishmania de acuerdo con la ubicación en el intestino del insecto, en la cual sitúa a las especies del complejo L. (V) braziliensis como de tipo peripilórico, es decir, que el desarrollo inicial de los parásitos ocurre en el intestino posterior con migración al intestino anterior antes de su transmisión por picadura.

En cuanto a los ensayos de transmisión del parásito a hámster sano por picadura (cuadro 2) se observó, en primer lugar, que el número de hembras que sobrevivieron a la oviposición y llegaron vivas al quinto o sexto día para realimentarse en hámsteres es bajo $(47,6$ y 18 para cada grupo), teniendo en cuenta que el número inicial de hembras que se alimentaron a través de membrana fue considerable $(102,127$ 


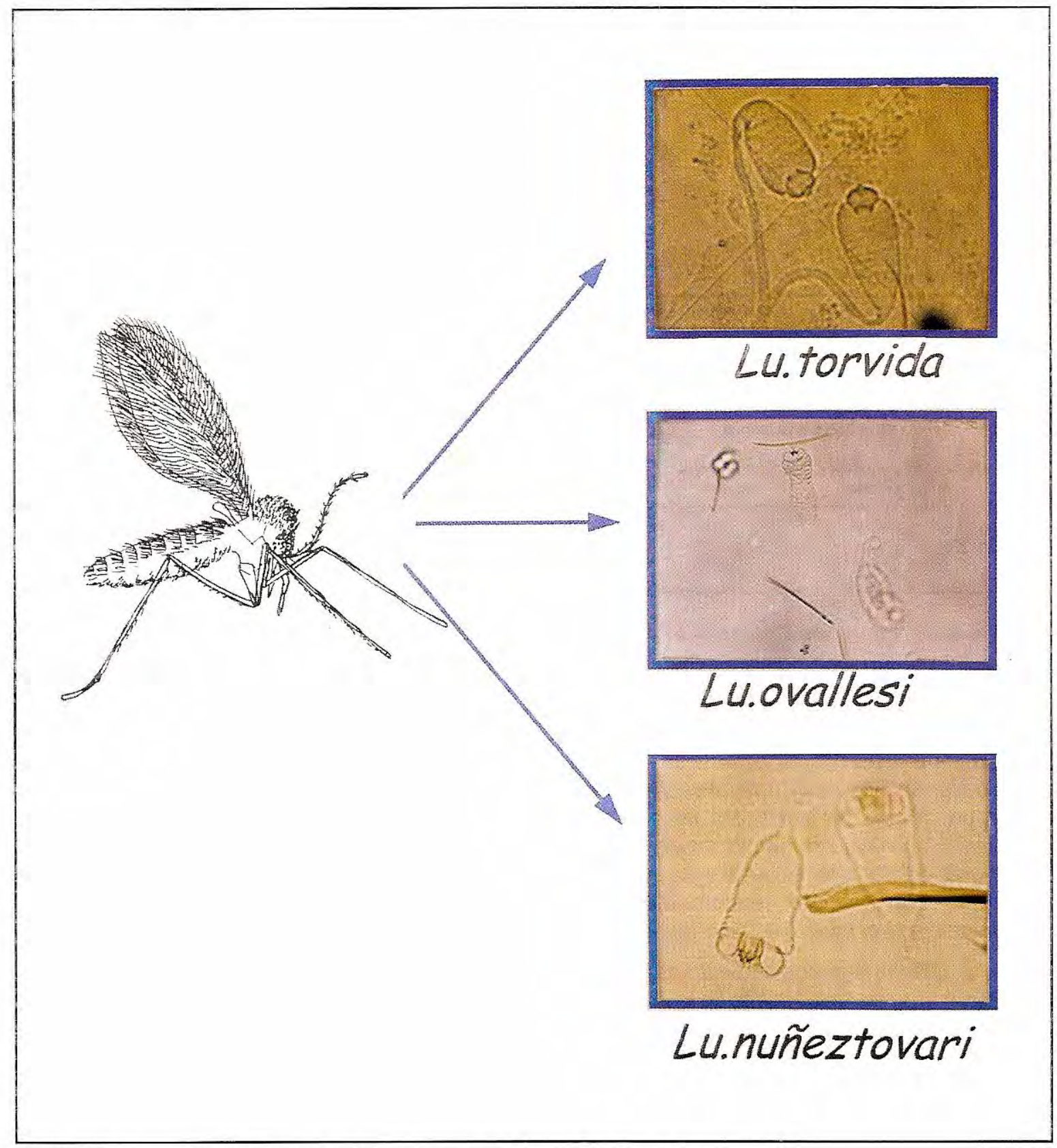

Figura 2. Espermatecas de las especies del grupo verrucarum presentes en el foco.

y 156); pero, es aún más bajo el número de hembras que se alimentaron por segunda vez del hámster sano (14, 4 y 2).

Al disecar estas hembras que picaron al hámster, se notó que la mitad de ellas, en cada grupo, fue positiva para la infección (cuadro 2).
En el tercer grupo, se presentó que las hembras infectadas que habían picado al hámster pertenecian a las tres especies del grupo verrucarum encontradas en el foco: 1 hembra de Lu. torvida, 4 de Lu. ovallesi y 2 de $L u$. nuñeztovari. En el cuarto grupo, 2 hembras 
Cuadro 1. Ensayos de infección experimental con Leishmania braziliensis de las especies de Lutzomyia del grupo verrucarum presentes en el foco endémico de Reventones, Cundinamarca.

\begin{tabular}{|c|c|c|c|c|c|c|c|}
\hline \multirow[t]{2}{*}{ Grupo } & \multirow[t]{2}{*}{ Epoca del año } & \multirow{2}{*}{$\begin{array}{c}\text { No. hembras } \\
\text { alimentadas } \\
\text { por membrana }\end{array}$} & \multirow{2}{*}{$\begin{array}{c}\text { No. hembras } \\
\text { disecadas } \\
\text { en total }\end{array}$} & \multicolumn{3}{|c|}{$\begin{array}{c}\text { No. hembras infectadas } / \mathrm{N} \text {. hembras disecadas } \\
\text { de cada especie }\end{array}$} & \multirow{2}{*}{$\begin{array}{l}\text { Porcentaje } \\
\text { de infección } \\
\text { general }\end{array}$} \\
\hline & & & & Lu. torvida & Lu. ovallesi & Lu. nuñeztovari & \\
\hline 1 & Septiembre,1997 & 29 & 21 & $11 / 20$ & $0 / 0$ & $1 / 1$ & $12 / 21=57,1 \%$ \\
\hline 2 & Noviembre,1997 & 69 & 67 & $15 / 46$ & $0 / 12$ & $1 / 9$ & $16 / 67=23,9 \%$ \\
\hline $3^{*}$ & Marzo, 1998 & 102 & 47 & $10 / 20$ & $18 / 21$ & $5 / 6$ & $33 / 47=70,2 \%$ \\
\hline $4^{*}$ & Julio, 1998 & 127 & 31 & $13 / 29$ & $1 / 1$ & $0 / 1$ & $14 / 31=45,2 \%$ \\
\hline $5^{*}$ & Septiembre.1998 & 156 & 19 & $4 / 15$ & $1 / 2$ & $1 / 2$ & $6 / 19=31,6 \%$ \\
\hline
\end{tabular}

*En estos grupos se realizaron, además, los ensayos de transmisión a hámster por picadura.

Cuadro 2. Ensayos de transmisión experimental de Leishmania braziliensis a hámster por picadura.

\begin{tabular}{|c|c|c|c|c|c|c|c|}
\hline \multirow[t]{2}{*}{ Grupo } & \multicolumn{2}{|c|}{ Intento de transmisión a hámster } & \multicolumn{3}{|c|}{$\begin{array}{l}\text { Composición de especies de hembras infectadas } \\
\text { que picaron en hámster }\end{array}$} & \multirow[t]{2}{*}{$\begin{array}{l}\text { Porcentaje } \\
\text { de infección }\end{array}$} & \multirow{2}{*}{$\begin{array}{c}\text { Análisis } \\
\text { Directo a } \\
\text { hámsteres }\end{array}$} \\
\hline & $\begin{array}{l}\text { N. hembras puestas a } \\
\text { re-alimentarse }\end{array}$ & $\begin{array}{l}\text { N. hembras que } \\
\text { picaron }\end{array}$ & Lu.torvida & Lu.ovallesi & Lu.nuñeztovari & & \\
\hline 3 & 47 & 14 & 1 & 4 & 2 & $7 / 14=50 \%$ & Negativo \\
\hline 4 & 6 & 4 & -..-.- & 2 & -.-.-. & $2 / 4=50 \%$ & Negativo \\
\hline 5 & 18 & 2 & 1 & --.-. & ----- & $1 / 2=50 \%$ & Negativo \\
\hline
\end{tabular}

infectadas de $L u$. ovallesi picaron al hámster y en el quinto grupo, una hembra infectada de $L u$. torvida picó al hámster.

Los tres hámsteres que fueron picados por hembras de Lutzomyia infectadas, fueron analizados en dos oportunidades bimensualmente por medio de improntas de exámenes directos y en ninguno se encontró la forma amastigota del parásito; sin embargo, se observó una moderada reacción leucocitaria, lo que podría indicar una infección poco manifiesta.

Rey et al. (33) no lograron aislar amastigotes de hámsteres dos meses después de la inoculación con promastigotes de L. braziliensis. Esta experiencia no es aplicable a la transmisión por picadura ya que la infectividad del parásito se intensifica en el intestino del vector. Aunque no se lograron visualizar amastigotes del parásito pasados cuatro meses, es posible que la infección sea tan baja que no puede ser detectada mediante examen directo.

\section{Discusión}

Las especies del complejo L. (V) braziliensis presentan dificultad para cultivarse y mantenerse en el laboratorio; al mismo tiempo, los ensayos de infección y transmisión experimental no son siempre exitosos (34). No ocurre lo mismo con las especies del complejo $L$. (Le) mexicana las cuales, bajo condiciones experimentales pueden desarrollarse y ser transmitidas por muchas especies del género Lutzomyia aún sin ser vectores naturales (35). Esta carencia de especificidad vector-parásito de las especies del complejo $L$. (Le) mexicana, no es el caso de la especie L. braziliensis, cuyos promastigotes experimentalmente parecen desarrollarse mejor en la especie de Lutzomyia que los transmite en la naturaleza. El desarrollo del parásito $L$. braziliensis en sus posibles vectores ha sido estudiado mediante infecciones experimentales en relativamente pocas especies de Lutzomyia, entre ellas: Lutzomyia intermedia (Lutz \& Neiva 1912), la cual es susceptible a la infección con L. braziliensis presentando colonización de parásitos en el intestino posterior y migración hacia el intestino anterior, siendo este un criterio importante para la incriminación de esta especie como vector de leishmaniasis cutánea en Rio de Janeiro (36). Lu. gomezi (Nitzulescu 1931), infectada experimentalmente a través de membrana de piel de pollo, registró un porcentaje de infección de $44 \%$ (37). Warburg et al. (10) 
infectaron con L. braziliensis: Lu. youngi (identificada erróneamente como Lu. townsendi) y Lu. columbiana (Ristorcelly \& Van Ty 1941), especies del grupo verrucarum, encontrando altos porcentajes de infección y considerando estas especies como posibles vectores de $L$. braziliensis en una plantación cafetera en Versalles, Valle del Cauca. Las anteriores especies de Lutzomyia son vectores sospechosos en sus áreas correspondientes, por lo que estas combinaciones vector-parásito son consideradas como asociaciones naturales. En su lugar, los intentos por infectar con $L$. braziliensis el flebótomo Lu. flaviscutellata (Mangabeira 1942), el cual no es vector natural, han sido infructuosos (4). Algo parecido ocurre con Lu. longipalpis (Lutz \& Neiva), que es vector comprobado de $L$. chagasi (el agente etiológico de la leishmaniasis visceral), pero al intentar infectar esta especie con L. braziliensis se presentan porcentajes de infección bajos y poca intensidad de parásitos, lo que muestra el pobre desarrollo de $L$. braziliensis en este inapropiado vector (10). Del mismo modo, en el Laboratorio de Entomología del INS se intentó infectar $L u$. longipalpis con una cepa de promastigotes de $L$. braziliensis; de 44 hembras alimentadas y disecadas 5 días después de la comida infectiva, ninguna presentó flagelados en su intestino lo que confirma la baja o nula susceptibilidad de esta especie a infectarse experimentalmente con L. braziliensis (38).

Aunque los parásitos del complejo $L$. (Le) mexicana han sido experimentalmente transmitidos por picadura de varias especies de flebótomos (39-41); los experimentos de transmisión con $L$. braziliensis han sido poco exitosos, aún cuando se usan vectores naturales (37). Esto podría deberse, en parte, a la dificultad de infectar altas proporciones de flebótomos con esta especie de Leishmania bajo condiciones experimentales (42) y, además, al bajo número de individuos que sobreviven a la oviposición y se alimentan por segunda vez (43). No existen muchos reportes de transmisión experimental exitosa de $L$. braziliensis a hámster (u otro hospedero) por picadura. Bajo condiciones experimentales, Lu. longiflocosa (del grupo verrucarum, serie townsendi), el flebótomo antropofílico dominante en un foco de $L$. braziliensis en Tello-Baraya, Huila logró transmitir el parásito a un hámster por picadura, aumentando las evidencias que indican que esta especie es el vector del parásito en dicha zona (13).

En este estudio, se pudo comprobar que las especies de Lutzomyia del grupo verrucarum presentes en el foco de leishmaniasis cutánea y mucocutánea en Reventones, Cundinamarca, $L u$. torvida, Lu. ovallesi y $L u$. nuñeztovari, son susceptibles de infectarse con $L$. braziliensis en condiciones experimentales, lo que indica que las tres especies podrían estar involucradas de una $u$ otra forma en el ciclo de transmisión del parásito en este foco endémico.

Lu. torvida fue la especie más abundante en la mayoría de los grupos y la más susceptible de infectarse con L. braziliensis, en la que se observó mayor grado de antropofilia y también es la especie más frecuente en el intra y peridomicilio (21) lo que aumenta el contacto con la población humana. Aunque la transmisión del parásito al hámster por picadura de Lu. torvida no se ha demostrado hasta el momento, vale la pena recordar que de las tres especies infectadas experimentalmente, Lu torvida fue la única especie en la que se observaron flagelados de cuerpo corto y alta movilidad en el intestino anterior, considerados como formas infectivas, lo cual según Warburg \& Schlein (44) implica la eficiencia en la transmisión de Leishmania. Las anteriores evidencias apuntan a que $L u$. torvida podría ser el vector primario de $L$. braziliensis en el foco de Reventones, Cundinamarca.

Lu. ovallesifue la segunda especie más frecuente y también fue susceptible a la infección con $L$. braziliensis. Esta especie perteneciente al grupo verrucarum, serie verrucarum, tiene una amplia distribución (24) y antecedentes vectoriales en varios países de Latinoamérica $(5,15-18)$. En el presente trabajo se notó que en el tercer grupo, correspondiente a la captura efectuada en marzo, Lu. ovallesi fue perceptiblemente más abundante que $L$ u. torvida, la cual predomina en los demás meses del año; del mismo modo, en este grupo, 
Lu. ovallesi presentó el más alto porcentaje de infección.

La especie Lu. nuñeztovari estuvo presente en todas las capturas y, aunque en una densidad muy baja, también llegó a infectarse en la mayoría de los grupos. Se ha registrado, a su vez, en proporciones bajas en el intra y peridomicilio en este foco (datos no publicados, Laboratorio de Entomología, INS), por lo cual podría estar comprometida en la transmisión de la enfermedad. En Colombia, esta especie se ha encontrado en densidades bajas en varios focos de $L$. braziliensis a lo largo de la Cordillera Oriental, como el de Arboledas, Norte de Santander (26), el de Tello-Baraya,Huila, y el de Reventones, Cundinamarca (25).

La participación de las tres especies en la transmisión del parásito debe analizarse con cuidado por que tal vez no todas participan en la misma forma. Los resultados de este estudio sugieren que $L u$. torvida es quizás la más comprometida en la transmisión del parásito al hombre; no obstante, es al parecer la más susceptible a los cambios ambientales, ya que en el intento de colonización de esta especie, realizado por Neira en 1997 (25), no se encontraron los requisitos mínimos para mantenerla en el laboratorio por varias generaciones consecutivas, pudiendo asumir que en el campo, cuando las condiciones microambientales como precipitación y humedad relativa no son favorables, las poblaciones de esta especie se ven afectadas. Esto coincide con las observaciones de abundancia estacional en las que especialmente en época seca la población de esta especie aumenta considerablemente alcanzando una abundancia de hasta 179 hembras/hora/trampa; por el contrario, en época lluviosa, ha llegado a un mínimo de 1 hembra/hora/trampa (datos no publicados, Laboratorio de Entomología, INS). Lu. ovallesi, aunque no es la especie más abundante, se ha logrado adaptar más fácilmente a las condiciones de laboratorio, encontrándose actualmente en la décimocuarta generación (45); por lo anterior y por su incursión en el intradomicilio, se cree que esta especie es la que mantiene el parásito a través del año, actuando como vector secundario o complementario. $L u$. nuñeztovari podría estar involucrada en el ciclo de transmisión, ya que es susceptible a la infección y se ha registrado en el peri e intradomicilio; esta especie probablemente ayuda a mantener la infección entre hospederosreservorios; no obstante, es muy poco lo que se conoce acerca de sus fuentes alimenticias. Aunque una variedad de esta especie, $L u$. nuñeztovari anglesi, es un vector importante de leishmaniasis cutánea en Bolivia, de tener algún papel en la transmisión del parásito en nuestro foco de interés sería muy secundario debido a su mínima abundancia.

Actualmente, en el Laboratorio de Entomología del INS, se realizan disecciones de flebótomos silvestres procedentes de este foco para búsqueda de flagelados de Leishmania, por observación directa de los intestinos al microscopio y utilización de técnicas de biología molecular.

Como conclusión, las tres especies del grupo verrucarum presentes en el foco de leishmaniasis cutánea y mucocutánea de Reventones, Cundinamarca, son susceptibles a la infección con L. braziliensis, y aunque en el presente estudio no se pudo constatar su capacidad para transmitir el parásito a un hospedero por picadura, es posible que estas especies estén involucradas en el ciclo de transmisión del parásito en este foco. Sin embargo, por su abundancia y hábito antropofílico y endofílico, $L u$. torvida probablemente es el vector más comprometido. Se considera que es importante continuar los estudios de capacidad y competencia vectorial que lleven a identificar la especie o especies vector del parásito en esta zona.

\section{Agradecimientos}

Al señor Marco F. Suárez por su colaboración en el trabajo de campo; a Olga Lucía Cabrera por su ayuda en la disección de los ejemplares. Por el apoyo financiero a Colciencias (código 124304-179-95), al Instituto Nacional de Salud y a la Universidad de la Salle.

\section{Referencias}

1. Feliciangeli D. La fauna flebotómica (Diptera, Psychodidae) en Venezuela. I. Taxonomía y distribución geográfica. Bol Dir Mal San Amb 1988;34:99-113. 
2. Botero D, Restrepo M. Parasitosis humanas. Segunda edición. Medellín: Ediciones Corporación para Investigaciones Biológicas; 1992:218.

3. Saravia G, Holguín AF, McMahon-Prattd, D’Alessandro A. Mucocutaneous leishmaniasis in Colombia: Leishmania braziliensis subspecies diversity. Am JTrop Med Hyg 1985;34:714-20.

4. Lainson R. The American leishmaniasis: some observations on their ecology and epidemiology. Trans Roy Soc Trop Med Hyg 1983;77:569-96.

5. Young DG, Morales A, Kreutzer RD, Alexander JB, Corredor A, Tesh RB, et al. Isolation of Leishmania braziliensis (Kinetoplastida: Trypanosomatidae) from cryopreserved Colombian sand flies (Díptera: Psychodidae). J Med Entomol 1987;24:587-9.

6. Montoya J, Travi B, Palma G, Jaramillo C. Report of an epidemic outbreak of tegumentary leishmaniasis in a coffee-growing area of Colombia. Mem Inst Oswaldo Cruz 1990;85:119-21.

7. Scorza JV, Castillo L, Rezzano S, Márquez M, Márquez C. El papel del cafeto en la endemicidad de la leishmaniasis cutánea en Venezuela. Bol Dir Mal San Amb 1985;25:82-8.

8. Scorza JV, Márquez M, Márquez C. Hallazgo de Lutzomyia townsendi (Ortiz, 1959) naturalmente infectada con Leishmania braziliensis, en el área suburbana de Trujillo, Venezuela. Bol Dir Mal San Amb 1984;24:21-8.

9. Scorza JV, Medina R, Pérez H, Hernández AG. Leishmaniasis. En: Chang KP, Bray RS, editors. $1^{\text {st }}$. ed. Armsterdam: Elsevier; 1985:283-96.

10. Warburg A, Montoya-Lerma J, Jaramillo C, Cruz-Ruiz AL, Ostrovska K. Leishmanias vector potencial of Lutzomyia spp. in Colombian coffee plantations. MedVet Entomol 1991;5:9-16.

11. Feliciangeli D, Arredondo C, Ward R. Phlebotomine sandflies in Venezuela: review of the verrucarum species group (III Part) of Lutzomyia (Díptera:Psychodidae) with description of a new species from Lara. J Med Entomol 1992;29:729-44.

12. Young DG, Duncan MA. Guide to identification and geographic distribution of Lutzomyia and sand flies in Mexico, West Indies, Central and South America (Diptera: Psychodidae). Mem Amer Entomol Inst 1994;54:1-881.

13. Santamaría E, Castillo M, Cárdenas R, Bello F, Ayala M, Ferro C. Transmisión experimental de Leishmania braziliensis a hámster por picadura de Lutzomyia longiflocosa (Díptera:Psychodidae) proveniente de un foco endémico en la zona cafetera colombiana. Médicas UIS 1998;12:279-84.

14. Travi BL, Vélez ID, Brutus L, Segura F, Jaramillo C, Montoya J. Lutzomyia evansian alternate vector of Leishmania chagasi in a Colombian focus of visceral leishmaniasis. Trans Roy Soc Trop Med Hyg 1990;84:6767.
15. Rowton ED, De Mata M, Rizzo N, Porter $\mathrm{CH}$, NavinTR. Isolation of Leishmania braziliensis from Lutzomyia ovallesi (Díptera:Psychodidae) in Guatemala. Am JTrop Med Hyg 1992;46:465-8.

16. Feliciangeli MD, Rodríguez N, Bravo A, Arias F, Guzınán B. Vectors of cutaneous leishmaniasis in northcentral Venezuela. Med Vet Entomol 1994;8:317-24.

17. Williams P. Phlebotomine sandflies and leishmaniasis in British Honduras (Belice). Trans Roy Soc Trop Med Hyg 1970;64:317-68.

18. Christensen HA, Fairchild GB, Herrer A, Johnson CM, Young DG, De Vásquez AM. The ecology of cutaneous leishmaniasis in the Republic of Panamá. J Med Entomol 1983;20:453-84.

19. Le Pont F, Mouchet J, Desjeux P. Leishmaniasis in Bolivia-VI. Observations on Lutzomyia nuñeztovari anglesi Le Pont \& Desjeux, 1984 the presumed vector of tegumentary leishmaniasis in theYungas focus. Mem Inst Oswaldo Cruz 1989;84:277-8.

20. Torrez M, López M, Le Pont F, Martínez E, Muñoz M, Hervas D, et al. Lutzomyia nuñeztovari anglesi (Diptera: Psychodidae) as a probable vector of Leishmania braziliensis in the Yungas,B olivia. Acta Tropica 1998;71:311-6.

21. Ferro C, Cabrera OL, Ayala M, Santamaría E, Castillo M, Neira M, et. al. Especies del grupo verrucarum (Diptera:Psychodidae) y su papel como vectores en dos focos de Leishmania braziliensis del centro de Colombia. XXV Congreso de la Sociedad Colombiana de Entomología SOCOLEN. Memorias 1998.p.41.

22. Ferro C, Morales A. Lista de las especies de flebótomos (Diptera: Psychodidae) de Colombia. Biomédica 1988;8:68-70.

23. Ferro C, Morales A. Flebótomos de Colombia: estudios realizados por el laboratorio de Entomología, 1965-1997. En: Toro G, Hernández CA, Raad J, editores. Instituto Nacional de Salud 1917-1997 Una historia, un compromiso. Santafé de Bogotá: Instituto Nacional de Salud; 1998:219-33.

24. Osorno E, Morales A, De Osorno F, Ferro C. Phlebotominae de Colombia (Díptera: Psychodidae) IX Distribución geográfica de especies de Brumptomyia Franca y Parrot, 1921 y Lutzomyia Franca, 1924 encontradas en Colombia. Rev Asoc Colomb Ciencias Exactas Físicas y Naturales 1972;53:45-68.

25. Neira M. Ciclo evolutivo de las especies antropofílicas más abundantes en dos focos de Leishmania braziliensis en Reventones, Anolaima (Cundinamarca) y en Tello (Huila). Tesis. Instituto Nacional de Salud-Universidad de la Salle. Santa Fe de Bogotá; 1998.

26. Alexander B, Ferro C, Young DG, Morales A, Tesh RB. Ecology of phlebotomine sand flies (Diptera: Psychodidae) in a focus of Leishmania (Viannia) braziliensis in northeastern Colombia. Mem Inst Oswaldo Cruz 1992;87:38795. 
27. Cárdenas R, Romo M, Santamaría E, Ferro C. Reconocimiento preliminar de las especies de flebótomos de un foco de leishmaniasis cutánea en el municipio de Planadas, Tolima. VI Encuentro Científico. Instituto Nacional de Salud. Biomédica 1999;19 (Suppl.1):117.

28. Killick-Kendrick R, Ward D. Ecology of Leishmania. Parasitology 1981;82:143-52.

29. Killick-Kendrick R. Studies and criteria for the incrimination of vectors and reservoir hosts of the leishmaniases. En: Walton BC, Wijeyaratne PM, Modabber F, editors. Research on control strategies for the leishmaniases. $1^{\text {st }}$. ed. Ottawa: IDRC manuscript report; 1988:184.

30. Bates PA. Infection of phlebotomine sandflies with Leishmania. In: Crampton JM, Beard CB, Louis C. The Molecular Biology of Insect Disease Vectors (a methods manual) 1st. ed. Gran Bretaña: Champan \& Hall; 1997:112-20.

31. Tesh RB, Modi GB. A simple method for experimental infection of phlebotomine sand flies with Leishmania. Am J Trop Med Hyg 1984;33:41-6.

32. Lainson R, Shaw J. The role of animals in the epidemiology in the South American leishmaniasis. In: LunsdenWH, editor. Biology of the Kinetoplastida. Vol II. London: Academic Press; 1979:9.

33. Rey JA,Travi BL, Valencia AZ, Saravia NG. Infectivity of the subspecies of the Leishmania braziliensis complex in vivo and in vitro. Am JTrop Med Hyg 1990;43:623-31.

34. Hommel M, Jaffe CL, Travi B, Milton G. Experimental models for leishmaniasis and for testing anti-leishmanial vaccines. Ann Trop Med Parasitol 1995(Suppl. 1):55-73.

35. Williams P, Coelho MV. Taxonomy and transmission of Leishmania. In: Lumsden WHR, Muller R Jr, Baker JR. Advances in Parasitology, Vol.16. New York: Academic Press; 1978:1-42.

36. Rangel EF, Travi B, Barbosa AF, Montoya J. Development of Colombian isolates of Leishmania (Vianna) panamensis, Leishmania (V) guyanensis and Leishmania (V) braziliensis in the sandfly Lutzomyia intermedia
(Lutz \& Neiva, 1912) under experimental conditions. Mem Inst Oswaldo Cruz 1993;88:513-15.

37. Jaramillo C, Travi B, Montoya T. Vector competence of some Neotropical sandflies for the Leishmania (Vianna) braziliensis complex. Med Vet Entomol 1994;8:1-7.

38. Castillo M, Santamaría E. Ensayo de infección experimental de Lutzomyia torvida y Lutzomyia Iongiflocosa con Leishmania braziliensis. Tesis. Instituto Nacional de Salud - Universidad de la Salle. Santafé de Bogotá; 1998.

39. Coelho MV, Falcao AR. Transmissao experimental de Leishmania. II Transmissao de amostra mexicana por picada de Phlebotomus longipalpis e de Phlebotomus renei. Rev Inst Med Trop Sao Paulo 1967;4:220-4.

40. Coelho MV, Falcao AR. Desenvolvimiento de especies do género Leishmania em espécies brasileiras de flebótomos do género Lutzomyia Franca, 1924. V. Infectividade de leptomonas evoluindo no flebótomo e experiencias de transmissao de leishmanioses. Rev Inst Med Trop Sao Paulo 1967; 9:367-73.

41. Scorza JV, Añez N. Transmisión experimental de Leishmania garnhami al hamster por la picadura de Lutzomyia townsendi. Rev Cub MedTrop 1984;36:139-45.

42. Franke ED, Rowton EO, McGreevy PB, Perkins PV. Detection and enumeration of Leishmania amastigotes and promastigotes in sandflies using agar plates, Abstr. Annu. Meet. Am Soc Trop Med Hyg 33 ${ }^{\text {rd; }}$; Baltimore 1984:179.

43. Killick-Kendrick R. Methods for the study of phlebotomine sandflies. In: Peters W. \& Killick-Kendrick R. The Leishmaniases in Biology and Medicine. Vol 1. 1a ed London: Academic Press; 1987:433-97.

44. Warburg GA, Schlein Y. The effect of post-blood meal nutrition of Phlebotomus papatasion the transmission of Leishmania major. Am J Trop Med Hyg 1986;35:926-30.

45. Cabrera OL, Neira M, Díaz A, Bello F, Suárez M, Ferro C. Ciclo de vida; cría y mantenimiento de ocho generaciones sucesivas de Lutzomyia ovallesi (Diptera: Psychodidae). XXV Congreso de la Sociedad Colombiana de Entomología SOCOLEN. Memorias 1998.p.44. 